\title{
Correction: Immune response and stromal changes in ductal carcinoma in situ of the breast are subtype dependent
}

\author{
Marie Colombe Agahozo • Pieter J. Westenend • Mieke R. van Bockstal (D) Tim Hansum • Jenny Giang • \\ Sanneke E. Matlung • Carolien H. M. van Deurzen
}

Published online: 29 October 2020

(c) The Author(s), under exclusive licence to United States \& Canadian Academy of Pathology 2020

Correction to: Modern Pathology

https://doi.org/10.1038/s41379-020-0553-9

There are some errors in the $P$ values reported in this paper. The correct results and table are shown below:

DCIS regression according to clinicopathological characteristics

We identified signs of DCIS regression in 30 out of 450 $(6.7 \%)$ patients. The association between DCIS regression and DCIS characteristics is reported in Table 2. Overall, DCIS regression was associated with a larger size $(P=$ $0.001)$, high grade $(P<0.0001)$, presence of comedonecrosis $(P=0.013)$, ER-PR-HER2 + IHC subtype $(P<$ $0.0001)$ and TIL-high DCIS $(P=0.006)$. After multivariate analysis, only the association between DCIS regression and ER-PR-HER2 + IHC subtype remained significant $(P=$ $0.001)$. 
Table 2 The association between DCIS regression and clinicopathological characteristics.

\begin{tabular}{|c|c|c|c|c|}
\hline & \multicolumn{2}{|c|}{ DCIS regression $(n=450)$} & \multirow{2}{*}{$\begin{array}{l}\text { Univariate } \\
P \text { value }\end{array}$} & \multirow{2}{*}{$\begin{array}{l}\text { Multivariate } \\
P \text { value }\end{array}$} \\
\hline & $\begin{array}{l}\text { Yes } \\
n(\%)\end{array}$ & $\begin{array}{l}\text { No } \\
n(\%)\end{array}$ & & \\
\hline Age at diagnosis (years) & & & 0.469 & - \\
\hline - Median (range) & $60.5(32.0-81.0)$ & $58.0(27.0-84.0)$ & & \\
\hline Size (missing $n=61)(\mathrm{cm})$ & & & 0.001 & 0.064 \\
\hline - Median (range) & $2.9(2.80-3.00)$ & $2.0(0.10-13.5)$ & & \\
\hline Growth pattern & & & 0.292 & - \\
\hline - Solid & $20(67)$ & $206(49)$ & & \\
\hline - Cribriform & $8(27)$ & $176(42)$ & & \\
\hline - Micropapillary & $2(7)$ & $33(8)$ & & \\
\hline - Papillary & $0(0)$ & $5(1)$ & & \\
\hline Grade & & & $<0.0001$ & 0.174 \\
\hline- Low & $0(0)$ & $58(14)$ & & \\
\hline - Intermediate & $4(13)$ & $152(36)$ & & \\
\hline - High & $26(87)$ & $210(50)$ & & \\
\hline Calcification & & & 0.835 & - \\
\hline - Absent & $9(30)$ & $118(28)$ & & \\
\hline - Present & $21(70)$ & $302(72)$ & & \\
\hline Comedonecrosis & & & 0.013 & 0.637 \\
\hline - Absent & $8(27)$ & $215(51)$ & & \\
\hline - Present & $22(73)$ & $205(49)$ & & \\
\hline $\begin{array}{l}\text { IHC DCIS subtype (missing } \\
n=18 \text { ) }\end{array}$ & & & $<0.0001$ & 0.001 \\
\hline - ER+PR+/-HER2- & $5(17)$ & $241(60)$ & & \\
\hline$-\mathrm{ER}+\mathrm{PR}+/$-HER $2+$ & $6(20)$ & $75(18)$ & & \\
\hline - ER-PR-HER2+ & $13(43)$ & $70(17)$ & & \\
\hline - ER-PR-HER2- & $6(20)$ & $16(4)$ & & \\
\hline Density of TILs & & & 0.006 & 0.818 \\
\hline -Low & $15(50)$ & $313(75)$ & & \\
\hline- High & $15(50)$ & $107(25)$ & & \\
\hline $\begin{array}{l}\text { Ipsilateral recurence } \\
\text { (missing } n=6 \text { ) }\end{array}$ & & & 1.000 & - \\
\hline- No & $28(97)$ & $400(96)$ & & \\
\hline- Yes & $1(3)$ & $15(4)$ & & \\
\hline
\end{tabular}

